Published in final edited form as:

Curr Treat Options Oncol. ; 19(6): 29. doi:10.1007/s11864-018-0543-z.

\title{
Promising New Agents for Colorectal Cancer
}

\author{
Satya Das, MD ${ }^{1}$, Kristen K. Ciombor, MD, MSCl${ }^{1}$, Sigurdis Haraldsdottir, MD, MSc $^{2}$, and \\ Richard M. Goldberg, MD ${ }^{3, *}$ \\ ${ }^{1}$ Division of Hematology and Oncology, Department of Internal Medicine, Vanderbilt University \\ Medical Center, 2220 Pierce Avenue, 777 Preston Research Building, Nashville, TN, 37232, USA \\ ${ }^{2}$ Division of Oncology, Department of Internal Medicine, Stanford University School of Medicine, \\ 875 Blake Wilbur Drive, Stanford, CA, 94305-6562, USA \\ ${ }^{3}$ West Virginia University Cancer Institute, P.O. Box 9300, 1801 HSS, 1 Medical Center Drive, \\ Morgantown, WV, 26506, USA
}

\section{Summary}

There are many exciting new therapies being developed for mCRC patients. Beyond adding to existing combination backbones of chemotherapy with oxaliplatin and irinotecan, subsets of mCRC patients have been identified who may benefit from novel treatments such as immune checkpoint blockade, HER2 inhibition, and MAPK blockade through targeting $R A S$ and $R A F$ based on genomic profiling from NGS. Other patients have demonstrated responses with developmental therapeutics including cytotoxic-conjugated antibodies targeting CEA, bi-specific antibodies drawing $\mathrm{CD} 3$ expressing $\mathrm{T}$ cells to tumor cells, metabolic modifiers to increase $\mathrm{T}$ cell proliferation at the tumor interface and epigenetic modulators preventing export of key tumor suppressor proteins. How to sequence these new options and determine where they fit into the framework of existing treatments remain major questions whose answers we hope to determine through ongoing clinical trials.

\section{Keywords}

Metastatic colorectal cancer; Therapeutics; Microsatellite instability; RAS; BRAF; HER2

\section{Introduction}

Outcomes of patients with mCRC have dramatically improved over the last decade. Overall survival (OS) has increased from 14.2 months with 5-fluorouracil (5FU)-based monotherapy

\footnotetext{
*Richard.goldberg@hsc.wvu.edu.

Conflict of Interest

Satya Das declares that he has no conflict of interest.

Kristen K. Ciombor has received funding for clinical trials paid to her institution from Pfizer, Boston Biomedical, MedImmune, Bayer, Boehringer Ingelheim, Bristol-Myers Squibb, Merck, Novartis, Incyte, Amgen, and Array BioPharma, and has also received reimbursement for travel expenses from Array BioPharma. Sigurdis Haraldsdottir declares that she has no conflict of interest.

Human and Animal Rights and Informed Consent

This article does not contain any studies with human or animal subjects performed by any of the authors.
} 
to near 30 months with targeted agent combinations with FOLFOX- or FOLFIRI-based chemotherapy [1]. Factors that further contribute to this OS gain include better surgical techniques that permit surgeons to resect oligometastatic disease (primarily in liver or lung), a more nuanced understanding of tumor biology (sidedness, $R A S$, and MSI status) and integration of biologic agents. Despite this progress, five-year survival rates remain $<15 \%$ for patients with stage IV disease [2]. The identification of new treatment options remains a priority for patients with advanced mCRC who have progressed through FOLFOX- and FOLFIRI-based therapies but who are still excellent candidates to receive further therapy based on their desire for additional treatment and a robust performance status. While singleagent therapy with either regorafenib or TAS-102 is approved in the USA as options after progression on initial infusion-based regimens, they each have only modest OS benefits compared with best supportive care [3, 4]. In the following paragraphs, we will focus on promising new therapeutics and treatment approaches that may positively influence outcomes for mCRC patients. Some of the most exciting of these include immunotherapy for patients with MSI-H/dMMR tumors, targeted agents for molecular subsets of mCRC patients (Table 1), CEA-seeking cytotoxic-conjugated antibodies and metabolic inhibitors which generate immunogenicity.

\section{Novel therapeutic options}

\section{Immunotherapy}

- $\quad$ Checkpoint inhibitors have recently garnered approval in MSI-H, chemotherapy refractory mCRC patients. Both single-agent nivolumab and pembrolizumab are now FDA-approved for mCRC patients after disease progression on oxaliplatinand irinotecan-based regimens. The Checkmate 142 study demonstrated the benefit of nivolumab in this setting and was a single-arm phase II study with ORR as the primary endpoint [5••]. In this study, 74 patients with recurrent or mCRC were treated with nivolumab $3 \mathrm{mg} / \mathrm{kg}(\mathrm{mg} / \mathrm{kg})$ every 2 weeks. The ORR was $31 \%$, while disease control rate (DCR) was $69 \%$. DCR for more than 4 months was achieved in $69 \%$ of patients.

- $\quad$ Pembrolizumab's efficacy was shown in the single-arm Keynote 164 phase II trial [6]. In this study, 61 patients with mCRC (90\% with at least two prior lines of therapy) were treated with pembrolizumab $200 \mathrm{mg}$ every 3 weeks. The primary endpoint for this study was overall response rate (ORR) and was $26 \%$. DCR was demonstrated in $51 \%$ of patients, and median duration of response was not reached.

- $\quad$ Given the success of single-agent therapy, immunotherapy combinations are also being explored in both MSI-H and microsatellite stable (MSS) refractory mCRC patients. In the Checkmate 142 study described above, other arms included nivolumab $3 \mathrm{mg} / \mathrm{kg}$ plus ipilimumab $1 \mathrm{mg} / \mathrm{kg} \times$ four doses (N3/I1) in MSI-H patients and nivolumab $1 \mathrm{mg} / \mathrm{kg}$ plus ipilimumab $3 \mathrm{mg} / \mathrm{kg}$ (N1/I3) and N3/I1 in MSS patients. In MSI-H patients, the combination resulted in ORR of 55\%, DCR of $80 \%$, and median PFS and OS which were not reached [7••]. In the MSS group, the N3/I1 combination produced an ORR of $0 \%$, PFS of 1.3 months and 
median OS of 3.73 months. The N1/13 combination produced an ORR of 10\%, PFS of 2.3 months, and OS of 11.3 months in the MSS patient cohort.

- Immunotherapy agents are also being combined with other agents in the mCRC setting. Hochster et al. presented findings from a phase $1 \mathrm{~b}$ study looking at the combination of the PD-L1 inhibitor atezolizumab plus bevacizumab in MSI-H $\mathrm{mCRC}$ patients [8]. A total of 10 patients were given both atezolizumab $1200 \mathrm{mg}$ and bevacizumab $15 \mathrm{mg} / \mathrm{kg}$ every 3 weeks. The combination was safe and produced an ORR of $30 \%$ with a DCR of $90 \%$. Median OS after 11 months of follow-up has not yet been reached.

- Given that only about $4 \%$ of patients with mCRC have tumors that are MSI-H, there naturally remains a great interest in translating immune-oncologic benefits to MSS patients. Bendell et al. presented findings from a phase I study looking at the combination of cobimetinib (20-60 mg oral daily) and atezolizumab (800 mg every 2 weeks) in MSS CRC patients [9]. Out of the 23 patients enrolled in the trial, 22 had tumors that were KRAS mutant. The combination was well tolerated at the maximum tolerated dose (MTD) of $60 \mathrm{mg}$ for cobimetinib and $800 \mathrm{mg}$ for atezolizumab with no observed DLTs. ORR was $17 \%$ and DCR was $39 \%$; three responses were ongoing at the time of data reporting. A phase III trial with this combination has completed accrual and the expectation is that results will be forthcoming soon.

- POLE and POLD-1 mutations in DNA polymerase genes can occur as germline [10] or tumor mutations in patients with CRC [11]. POLE/POLD-1 mutations invariably are associated with a high tumor mutational burden and are usually not associated with a MSI-high state. They appear to occur in 1-3\% of CRC although the incidence may be lower in mCRC. Case reports have shown responses to pembrolizumab [12].

\section{BRAF V600E inhibition}

- $\quad$ Among the 5-10\% of mCRC patients with BRAF mutations, 90\% have BRAF V600E mutations. Patients who have tumors with this specific BRAF mutation generally have a poorer prognosis. Unfortunately, unlike in BRAF V600E mutant patients with melanoma where BRAF inhibition with single agents such as dabrafenib or vemurafenib produces significant benefits in ORR, PFS and OS, this approach has not been successful in CRC patients. Sundar et al. demonstrated that melanoma cells exhibit minimal rebound in EGFR signaling, while CRC cell lines demonstrate rapid negative feedback upregulation and signaling through EGFR when exposed to BRAF inhibitors [13].

- Several exploratory approaches that combine agents which target the RAF pathway have shown promise in these patients, including triplet therapy with BRAF, MEK and EGFR inhibition, and BRAF and EGFR inhibitors plus irinotecan.

- $\quad$ Atreya et al. first reported results from the triplet combination in 2015 [14]. A total of $35 \mathrm{mCRC}$ patients with BRAF V600E mutations received dabrafenib 150 
$\mathrm{mg}$ twice daily, trametinib $2 \mathrm{mg}$ daily and panitumumab $6 \mathrm{mg} / \mathrm{kg}$ every 2 weeks. ORR for this combination was $26 \%$ and DCR was $83 \%$; median duration of response was 5.4 months. In contrast, in patients on the study who received dabrafenib twice daily and panitumumab every 2 weeks, ORR was $10 \%$. Phosphorylated ERK levels between pre-treatment and on-treatment biopsies were more profoundly reduced in the triplet regimen than in the doublet regimen. This suggests that the strategy of hitting multiple targets within the pathway may overcome drug resistance in this group of patients.

- The combination of vemurafenib, cetuximab, and irinotecan was initially reported by Kopetz et al. in a phase II trial in 2017 [15]. In this study, mCRC patients whose tumors harbored BRAF V600E mutations and who had received up to two prior lines of therapy were randomized to irinotecan $180 \mathrm{mg} / \mathrm{m}^{2}$ every 14 days, cetuximab $500 \mathrm{mg} / \mathrm{m}^{2}$ every 14 days and vemurafenib $960 \mathrm{mg}$ twice daily, or irinotecan and cetuximab at the same dosages. Median PFS in the triplet arm was 4.4 months compared to 2.0 months in the doublet arm. ORR was $16 \%$ and DCR was $67 \%$ in the triplet arm compared to 4 and $22 \%$, respectively, in the doublet arm. A total of $50 \%$ of patients in the doublet arm crossed over at the time of progression.

- The BEACON-CRC trial is an ongoing three arm phase III study assessing the efficacy of encorafenib $300 \mathrm{mg}$ daily, binimetinib $45 \mathrm{mg}$ twice daily, and cetuximab $250 \mathrm{mg} / \mathrm{m}^{2}$ weekly versus encorafenib and cetuximab versus irinotecan or FOLFIRI and cetuximab in patients with BRAF V600E mutant CRC. Early results from the trial were presented in abstract form in 2018 by Van Cutsem et al. [16]. Of the initial 29 patients treated on the encorafenib, binimetinib, and cetuximab arm, ORR was $41 \%$, DCR was $72 \%$, and median time on treatment was 5.6 months.

- Another triplet combination that has shown promise in BRAF V600E mutant $\mathrm{mCRC}$ patients is the combination of encorafenib $200 \mathrm{mg}$ daily, cetuximab 500 $\mathrm{mg} / \mathrm{m}^{2}$ every 2 weeks and the PI3K inhibitor alpelisib $300 \mathrm{mg}$ daily [17]. Tabernero et al. reported findings from a randomized phase II study in which 102 BRAF V600E mutant CRC patients were randomized in the third line setting to the triplet regimen versus encorafenib and cetuximab. Median PFS and ORR with the triplet regimen was 5.4 months and $27 \%$ compared to 4.2 months and $22 \%$, respectively, with the doublet.

\section{HER2 inhibition}

- HER2 amplification occurs in 3-5\% of colorectal tumors. In the preclinical setting, HER2-amplified xenografts were not inhibited by single-agent trastuzumab or lapatinib. The combination, however, appears to overcome singleagent tumor resistance. These observations have given rise to three therapeutic trials under the HERACLES moniker [18].

- In the proof-of-concept phase II HERACLES study from Sartore-Bianchi et al., 27 heavily pre-treated patients whose tumors were HER2 amplified (IHC 2+ 
with FISH confirmation, or 3+ by IHC) and who had previously been treated with an average of four prior lines of therapy were treated with oral lapatinib $1000 \mathrm{mg}$ daily and trastuzumab $2 \mathrm{mg} / \mathrm{kg}$ weekly [19]. Seventy-four percent of patients achieved DCR, while 30\% of patients achieved ORR. In the B-cohort of the HERACLES trial, patients with HER2-amplified CRC were treated with pertuzumab and T-DM1. Preliminary results suggest seven of eight evaluable patients have received clinical benefit.

- The MOUNTAINEER study is an ongoing single arm phase II study of tucatinib, an oral selective small molecule inhibitor of HER2, $300 \mathrm{mg}$ daily combined with trastuzumab $6 \mathrm{mg} / \mathrm{kg}$ every 3 weeks in patients with HER2-amplified (IHC 3+ or amplified by FISH) mCRC. Strickler et al. presented the framework of the trial in 2017 but results have not yet been reported [20].

\section{Tropomyosin receptor kinases fusion inhibition}

- $\quad$ Tropomyosin receptor kinases (TRK) proteins are encoded by the neurotrophic receptor tyrosine kinase genes NTRK1, NTRK2, and NTRK3. Recurrent TRK fusions with different upstream partner proteins create oncogene addicted cancers across diverse sites; these mutations are found in $0.2-2.4 \%$ of $\mathrm{mCRC}$ patients. Inhibition of tumors expressing these fusion proteins with selective small molecule inhibitors such as larotrectinib has become an area of interest due to remarkable responses seen with monotherapy.

- $\quad$ Results with larotrectinib were recently reported as an aggregate of three trials (a phase I, phase I/II and phase II) by Drilon et al. [21]. Fifty-five patients, four of whom had mCRC, with 17 unique TRK fusions confirmed by NGS were treated with larotrectinib $100 \mathrm{mg}$ twice daily in adults or $100 \mathrm{mg} / \mathrm{m}^{2}$ in pediatric patients with a BSA G $1 \mathrm{mg} / \mathrm{m}^{2}$ in the phase II studies. ORR was $75 \%$ with $13 \%$ of patients demonstrating CR and 62\% demonstrating PR; DCR was $88 \%$. Median duration of response after 8.3 months of follow-up has not been reached, while median PFS after 9.9 months of follow-up has not been reached. Response was reported on three of the four CRC patients; two achieved a partial response (PR) while one achieved stable disease (SD).

\section{Antibody-conjugated chemotherapy agents}

- Labetuzumab govitecan (IMMU-130) is an antibody conjugated chemotherapy agent which targets carcinoembryonic antigen-related cell adhesion molecule 5 (CEACAM). This molecule is expressed in over $80 \%$ of colorectal cancers. Upon binding to its target, the agent releases $\mathrm{SN}-38$, the active metabolite of irinotecan, into the cancer cell, creating targeted cytotoxicity. Furthermore, much higher doses of SN-38 are deposited into the tumor with reduced serum levels of glucuronidated $\mathrm{SN}-38$, a factor that can reduce the drug's propensity to cause diarrhea [22].

- $\quad$ Results from a phase I/II study with IMMU-130 were recently published by Dotan et al. [23•]. In this study, $86 \mathrm{mCRC}$ patients who had been exposed to a 
median of five prior therapies received labetuzumab govitecan. Four doseexpanded cohorts $(8 \mathrm{mg} / \mathrm{kg}$ weekly, $10 \mathrm{mg} / \mathrm{kg}$ weekly, $4 \mathrm{mg} / \mathrm{kg}$ bi-weekly, and 6 $\mathrm{mg} / \mathrm{kg}$ bi-weekly every two out of 3 weeks) were determined based on DLTs experienced during the dose escalation portion of the study. In this study, 50\% of all patients achieved DCR, with most patients demonstrating SD with the treatment. Thirty-three of patients had SD for at least 4 months.

\section{Bi-specific T cell engagers}

- $\quad$ CEA-CD3 TCB (T cell bispecific) (RG7802) is an antibody which binds to both $\mathrm{CEA}$ on tumor cells and the $\mathrm{CD} 3$ receptor on $\mathrm{T}$ cells. Its Fc region was designed specifically to reduce inadvertent binding to complement elements or Fc gamma receptors. This therapeutic strategy is based on the premise that increasing $\mathrm{T}$ cell infiltration into tumors improves clinical outcome of patients with multiple tumor types, including CRC [24]. Combination of this agent with PD-1 or PD-L1 inhibitors in tumors that are not MSI-H is an appealing therapeutic strategy.

- In two separate ongoing studies reported in 2017, mCRC patients were given RG7802 alone weekly or in combination with the PD-L1 inhibitor atezolizumab at a dose of $1200 \mathrm{mg}$ every 3 weeks [25•]. In the patients receiving RG7802 monotherapy, doses up to $600 \mathrm{mg}$ were tolerated with minimal dose-limiting toxicities (DLT). Single-agent DCR in mCRC patients was 9\%. In the combination study, doses up to $160 \mathrm{mg}$ of RG7802 were tolerated well with only one noted DLT of liver function test elevation. Increased efficacy was suggested with a DCR of $25 \%$ in mCRC patients. Updated data from both trials are pending.

\section{Targeting RAS mutant disease}

- The prognostic influence of RAS mutations in mCRC patients is controversial, although some studies suggest worse outcomes in patients with RAS mutated tumors. However, RAS mutations are clearly predictive biomarkers for resistance to anti-EGFR therapies. The prevalence of RAS mutations in CRC patients has been estimated as high as $40-50 \%$, and therefore this family of genes is a target of high interest [26].

- $\quad$ RAS targeting agents have been very difficult to develop due to cross talk and redundancy within the MAPK pathway that appears to allow tumors to evade susceptibility to single agents. Many compounds targeting MAPK escape pathway mediators such as PI3K and mTOR have failed to show efficacy in early phase settings [27] (Fig. 1).

- $\quad$ MEK inhibition plus BCL-XL inhibition with navitoclax (ABT-263) and CDK4/6 inhibition represent approaches that appear promising preclinically. Corcoran et al. identified the anti-apoptotic BCL-XL gene as a potential target to combine with MEK inhibition in KRAS mutant CRC cell lines through shRNA screening [28]. Navitoclax plus selumetinib caused significantly higher levels of apoptosis than either agent alone in RAS mutant cell lines than in RAS wild-type 
cell lines. Navitoclax plus the MEK inhibitor trametinib is being investigated in a phase I/II trial in patients with KRAS or NRAS mutant advanced solid tumors [NCT02079740].

- $\quad$ The CDK4/6 inhibitor palbociclib has been investigated as monotherapy in a phase II trial in CRC patients with tumors harboring KRAS mutations [29]. From O'Hara et al., 36 patients with KRAS mutant tumors were screened for $\mathrm{Rb}$ expression and 35 were found to be Rb positive. Of these patients, 15 were treated with palbociclib at $125 \mathrm{mg}$ for 21 out of every 28 days. In this study, DCR was 33\%. Nine patients underwent pre-treatment and post-cycle one FLTPET scans as an imaging correlate, and six of the nine demonstrated metabolic response as measured by decrease uptake in visualized lesions.

- $\quad$ Reolysin $®$ is a ubiquitous reovirus which selectively replicates in KRAS mutant cells, resulting in lysis and cell death. Single-agent Reolysin ${ }^{\circledR}$ was shown to be safe in KRAS mutant CRC patients and prompted a subsequent chemotherapy combination trial [30]. Ocean et al. treated 21 CRC patients whose tumors had KRAS mutations with FOLFIRI plus Reolysin ${ }^{\circledR}$ [31]. Two patients experienced a DLT of neutropenia and thus the RP2D was determined to be $150 \mathrm{mg} / \mathrm{m}^{2}$ of irinotecan in conjunction with Reolysin ${ }^{\circledR} 3 \times 10^{10}$ tissue culture infectious dose (TCID50) D1-D5 every 28 days. Median PFS in FOLFIRI-naïve patients (9/21) was 7.4 months, while median PFS was not reached in prior FOLFIRI (12/21)treated patients. The results from a randomized phase II study of FOLFOX plus bevacizumab plus Reolysin ${ }^{\circledR}$ versus FOLFOX plus bevacizumab alone are pending [NCT01622543].

\section{Modified fluoropyrimidine (NUC-3373)}

- Fluoropyrimidines have been long been a cornerstone of therapy for mCRC patients, both as monotherapy or in combination with other agents. Resistance to 5FU and capecitabine is stimulated by thymidine kinase inhibition, thymidine phosphorylase overexpression, and membrane transporter inhibition [31].

- $\quad$ NUC-3373 is a novel fluoropyrimidine whose levels are unaffected by the enzyme dihydropyrimidine dehydrogenase (DPD) in vitro. Furthermore, the NUC-3373 compound generated 363-fold higher concentrations of the toxic metabolite F-dUMP intracellularly. In CRC xenografts, NUC-3373 delivered at equimolar doses to $5 \mathrm{FU}$ generated significantly greater tumor growth inhibition than $5 \mathrm{FU}(47 \%$ versus $25 \%)$ and generated lower levels of the toxic metabolite dihydroflurouracil [32].

- $\quad$ These preclinical findings inspired a phase I trial looking at NUC-3373 in patients with refractory advanced solid tumors. Interim PK and PD data from this trial was presented in 2017 at the European Society for Medical Oncology (ESMO) meeting and demonstrated rapid intra-cellular concentrations of the active metabolite F-dUMP without the generation of key toxic metabolites [NCT02723240]. 


\section{Glutaminase inhibitor (CB-839)}

- Mitochondrial glutaminase (GLS), the enzyme responsible for converting glutamine to glutamate, is overexpressed in many tumor types, including CRC. GLS expression has been correlated with adverse clinicopathologic features in CRC patients and was found to be associated with higher $\mathrm{T}$ stage and the presence of distant metastases. In vitro inhibition of GLS in the colon cancer cell lines HT29 and SW480 resulted in growth suppression and increased apoptosis [33]. Additionally, because glutamine can stimulate $\mathrm{T}$ cell activation, raising its levels through GLS inhibition can promote late stages of T cell activation resulting in an immunogenic microenvironment [34].

- $\quad$ CB-839 is a potent and selective inhibitor of glutaminase and is being explored in a myriad of early phase studies. At Case Western, a study in metastatic CRC patients with tumors that are fluoropyrimidine-resistant are receiving CB-839 plus capecitabine. The phase I portion of the study is a traditional dose escalation study, while the phase II portion is a dose expansion in patients with tumors that exhibit a PIK3CA mutation [NCT02861300]. At Vanderbilt, CB-839 plus panitumumab and irinotecan is being explored in a phase I study in patients with RAS WT tumors. The phase II portion of this trial will look at CB-839 plus panitumumab in patients previously treated with EGFR inhibitors [NCT03263429].

\section{SINE inhibitors}

- $\quad$ SINE inhibitors represent a class of agents which are selective inhibitors of nuclear export proteins. Tumor suppressor genes can be inactivated by export proteins, rendering them unable to carry out their normal regulatory function in the nuclei of cancer cells. Furthermore, resistance to DNA damaging agents such as irinotecan can be mediated through nuclear export proteins such as CRM1 [35].

- $\quad$ Preclinically, treatment of cell lines with the SINE inhibitor KPT-251 demonstrated nuclear sequestration of the protein topoisomerase 1 (Top1). Top1 is the target enzyme of irinotecan. Dramatic synergism between KPT-251 and CPT-11 was demonstrated in cell lines from both topoisomerase sensitive and refractory patients, suggesting the mechanism of action of KPT-251 stems from inhibition of CRM1 mediated Top1 export [36].

- $\quad$ A phase I expansion trial of KPT-330 (selinexor), an XPO-1 nuclear export protein inhibitor, in CRC patients was reported in abstract form in 2014. From Mau-Sorensen et al., $27 \mathrm{mCRC}$ patients with an average of four prior lines of therapy were treated with KPT-330. In this trial, 28\% of patients achieved DCR, with three patients demonstrating SD for at least 6 months. Pre- and posttreatment biopsies from patients demonstrated the restoration of nuclear localization of tumor suppressor proteins [37]. 
- A second-generation XPO-1 inhibitor, KPT-8602, is being investigated in a phase $\mathrm{I} / \mathrm{II}$ trial in patients with refractory solid tumors, including CRC

[NCT02649790].

\section{WNT pathway inhibitors}

- The WNT pathway is dysregulated in many CRCs and as such, is a potentially promising therapeutic target. A growing body of research links this pathway with cancer cells expressing a CRC stem cell phenotype. The stem cell phenotype is associated with tumor chemoresistance through the upregulation of transporters involved in drug efflux [38].

- $\quad$ There are numerous ongoing clinical trials targeting various members of this pathway, including B-catenin, which is a mediator of the classical WNT pathway. Some of these inhibitors include Foxy-5, LGK-974, vantictumab, PRI-724, and BBI-608 [39].

- Napabucasin (BBI-608) represents a first-in-class inhibitor of STAT-driven gene transcription in CRC stem cells. Preclinically, it has also demonstrated ability to sensitize CRC cell lines to chemotherapeutic agents such as irinotecan and 5-FU. The agent has exhibited promise in mCRC model systems along with cell cultures of tumors derived from other gastrointestinal malignancies [40].

- In the initial phase I study, napabucasin was well tolerated, with a recommended phase II dose (R2PD) of $500 \mathrm{mg}$ BID. Findings from a phase II expansion cohort of $K R A S$ WT colorectal cancer patients were reported in 2017 in abstract form [41]. Out of 48 patients who received napabucasin plus bi-weekly panitumumab ( $85 \%$ who had received at least three lines of therapy), DCR was achieved in $52 \%$. In 31 patients previously treated with anti-EGFR directed therapy, the DCR was $48 \%$ compared with $59 \%$ in anti-EGFR treatment-naïve patients. Another phase I/II study from Bendell et al. looked at the combination of FOLFIRI \pm bevacizumab and napabucasin in pre-treated $\mathrm{mCRC}$ patients [42•]. Of 82 enrolled patients, 32 had already manifested progressive disease on FOLFIRI \pm bevacizumab. There were no DLTs and ORR was $22 \%$, while DCR was $83 \%$. In 27 patients who were previously exposed to FOLFIRI, DCR was $81 \%$ and ORR was $21 \%$. This was nearly identical to patients who were FOLFIRI-naïve. The promising findings from this study have spawned the CanStem $303 \mathrm{C}$ trial, a phase III randomized study of napabucasin plus FOLFIRI versus FOLFIRI alone in second line mCRC patients. Enrollment is currently underway [43].

\section{New approaches with old agents}

- The results from the REVERCE study were presented in 2018 by Shitara et al. [44•]. In this trial, 101 patients with $K R A S$ exon two wild-type colorectal adenocarcinomas with disease progression on prior 5FU-, oxaliplatin- and irinotecan-based therapies were randomized to regorafenib followed by cetuximab with or without irinotecan or cetuximab with or without irinotecan followed by regorafenib. The primary endpoint was OS, while secondary 
endpoints included PFS with first (PFS1) and second (PFS2) treatments. Sequential treatment was completed in $86 \%$ of patients and after 29 months of follow-up, median OS in the therapy arm where regorafenib was initiated first was 17.4 months compared to 11.6 months in the cetuximab first arm (HR 0.61). There was no statistically significant difference between PFS1 between the arms; however, interestingly there was a difference in PFS2 with a HR of 0.29 for the regorafenib first treatment arm. No statistically significant differences in adverse events were seen in either arm.

- $\quad$ Results from the ReDOS study were also presented in 2018 by Bekaii-Saab et al. [45]. In this randomized phase II study, mCRC patients were randomized between a weekly dose escalation arm $(80 \mathrm{mg}$ for 1 week, then dose escalation as tolerated of $40 \mathrm{mg}$ per week up to $160 \mathrm{mg}$; final tolerated dose given for 21 of 28 days per cycle) and a standard dose arm (160 mg daily for 21 of 28 days per cycle) of regorafenib. The primary endpoint was the percentage of patients who completed 2 cycles of therapy and started on a third cycle. Secondary endpoints included OS and PFS. Forty-three percent of patients in the experimental arm versus $25 \%$ of patients in the standard treatment arm were able to receive a third cycle of regorafenib ( $p \mathrm{G}$.028). Median OS was 9 months in the experimental arm and 5.9 months in the standard treatment arm (not statistically significant). Overall rates for $\mathrm{G} 3 / \mathrm{G} 4$ toxicity were more favorable in patients treated with the experimental regimen with lower rates of hand-foot syndrome, fatigue, and hypertension. This trial suggests rapid weekly dose escalation is the optimal way to administer regorafenib for $\mathrm{mCRC}$ patients.

\section{Acknowledgments}

Richard M. Goldberg has received research funding through a grant from Merck, and has received compensation from Merck, Merck KGA, and Taiho for service as a consultant.

\section{References and Recommended Reading}

Papers of particular interest, published recently, have been highlighted as:

- Of importance

$\bullet$ Of major importance

1. Kopetz S, Chang GJ, Overman MJ, Eng C, Sargent DJ, Larson DW, et al. Improved survival in metastatic colorectal cancer is associated with adoption of hepatic resection and improved chemotherapy. J Clin Oncol 2009;27:3677-83. [PubMed: 19470929]

2. SEER Database 2018 https://seer.cancer.gov/statfacts/html/colorect.html.

3. Mayer R, Van Cutsem E, Falcone A, et al. Randomized trial of TAS-102 for refractory metastatic colorectal cancer. N Engl J Med 2015;372:1909-19. [PubMed: 25970050]

4. Grothey A, Van Cutsem E, Sobrero A, et al. Regorafenib monotherapy for previously treated metastatic colorectal cancer (CORRECT): an international, multicentre, randomised, placebocontrolled, phase 3 trial. Lancet 2013;381(9863):303-12. [PubMed: 23177514]

5••. Overman M, McDermott R, Leach J, et al. Nivolumab in patients with metastatic DNA mismatch repairdeficient or microsatellite instability-high colorectal cancer (CheckMate 142): an openlabel, multicentre, phase 2 study. Lancet Oncol 2017;18(9):1182-91. [PubMed: 28734759] A proof of concept trial which led to the approval of nivolumab in MSI-high cancers after standard 
chemotherapy approaches, and furthermore, in mCRC patients post exposure to oxaliplatin and irinotecan. Beyond the ORR demonstrated with the single-agent therapy, the duration of response demonstrated by responding patients was striking.

6. Diaz L, Marabelle A, Kim TW, et al. Efficacy of pembrolizumab in phase 2 KEYNOTE-164 and KEYNOTE-158 studies of microsatellite instability high cancers. Ann Oncol 2017;28(5): $\mathrm{mdx} 367.020$.

7••. Overman M, Lonardi S, Yeung K, et al. Durable clinical benefit with nivolumab plus ipilimumab in dna mismatch repair-deficient/microsatellite instability-high metastatic colorectal cancer. J Clin Oncol 2018;36(8):773-9. [PubMed: 29355075] An analysis which builds upon the effectiveness of single-agent nivolumab in patients with MSI-high tumors and demonstrates that these patients have an even greater ORR and DCR with the combination of nivolumab and ipilimumab than with single-agent checkpoint inhibitor therapy.

8. Hochster H, Bendell J, Cleary J, et al. Efficacy and safety of atezolizumab (atezo) and bevacizumab (bev) in a phase Ib study of microsatellite instability (MSI)-high metastatic colorectal cancer (mCRC). J Clin Oncol 2017;35(4):S673.

9. Bendell J, Kim TW, Goh B, et al. Clinical activity and safety of cobimetinib (cobi) and atezolizumab in colorectal cancer (CRC). J Clin Oncol 2016;34(15):S3502.

10. Palles C, Cazier JB, Howarth K, et al. Germline mutations affecting the proofreading domains of POLE and POLD1 predispose to colorectal adenomas and carcinomas. Nat Genet 2013;45:13644. [PubMed: 23263490]

11. TCGA. Comprehensive molecular characterization of human colon and rectal cancer. Nature 2012;487:330-7. [PubMed: 22810696]

12. Gong J, Wang C, Lee PP, Chu P, Fakih M. Response to PD-1 blockade in microsatellite stable metastatic colorectal cancer harboring a POLE mutation. JNCCN 2017;15:142-7. [PubMed: 28188185]

13. Sundar R, Hong D, Kopetz S, et al. Targeting BRAF-mutant colorectal cancer: progress in combination strategies. Cancer Discov 2017;7(6):558-60. [PubMed: 28576843]

14. Atreya CE, Van Cutsem E, Bendell J, et al. Updated efficacy of the MEK inhibitor trametinib (T), BRAF inhibitor dabrafenib (D), and anti-EGFR antibody panitumumab (P) in patients (pts) with BRAF V600E mutated (BRAFm) metastatic colorectal cancer (mCRC). J Clin Oncol 2015;33(15):S103.

15. Kopetz S, Desai J, Chan E, Hecht JR, O’Dwyer PJ, Maru D, et al. Phase II pilot study of vemurafenib in patients with metastatic BRAF-mutated colorectal cancer. J Clin Oncol 2015;33:4032-8. [PubMed: 26460303] A proof of concept trial which highlights the potential ability of BBI-608 to resensitize mCRC patients to chemotherapy backbones (FOLFIRI) that they may have progressed on previously.

16. Van Cutsem E, Cuyle P, Huijberts S, et al. BEACON CRC study safety lead-in (SLI) in patients with BRAFV600E metastatic colorectal cancer (mCRC): efficacy and tumor markers. J Clin Oncol 2018;36(4):S627.

17. Tabernero J, Van Geel R, Guren TK, et al. Phase 2 results: encorafenib (ENCO) and cetuximab (CETUX) with or without alpelisib (ALP) in patients with advanced BRAF-mutant colorectal cancer (BRAFm CRC). J Clin Oncol 2016;34(15):S3544.

18. Seo A, Kwak Y, Kim DK, et al. HER2 status in colorectal cancer: its clinical significance and the relationship between HER2 gene amplification and expression. PLoS One 2014;9(5):e98528. [PubMed: 24879338]

19. Sartore-Bianchi A, Trusolino L, Martino C, Bencardino K, Lonardi S, Bergamo F, et al. Dualtargeted therapy with trastuzumab and lapatinib in treatment-refractory, KRAS codon 12/13 wildtype, HER2-positive metastatic colorectal cancer (HERACLES): a proof-of- concept, multicentre, open-label, phase 2 trial. Lancet Oncol 2016;17(6):738-46. [PubMed: 27108243]

20. Strickler J, Niedzwiecki D, Zemla T, et al. A phase II, open label study of tucatinib (ONT-380) combined with trastuzumab in patients with HER2+ metastatic colorectal cancer (mCRC) (MOUNTAINEER). J Clin Oncol 2017;35(15):S3624.

21. Drilon A, Laetsch S, Kummar S, et al. Efficacy of Larotrectinib in TRK fusion-positive cancers in adults and children. N Engl J Med 2018;378:731-9. [PubMed: 29466156] 
22. Govindam S, Cardillo T, Rossi E, et al. IMMU-130, a unique antibody-drug conjugate (ADC) of SN-38 targeting CEACAM5 antigen: preclinical basis for clinical activity in metastatic colorectal cancer (mCRC). J Clin Oncol 2015;33(3):S625.

23•. Dotan E, Cohen S, Starodub A, et al. Phase I/II trial of labetuzumab govitecan (Anti-CEACAM5/ SN-38 antibody-drug conjugate) in patients with refractory or relapsing metastatic colorectal cancer. J Clin Oncol 2017;35(29):3338-46. [PubMed: 28817371] This study suggests a role for CEA directed antibody-drug conjugates in colorectal cancer as the agent appears to have singleagent activity in heavily pre-treated patients. Furthermore, the trial raises possibilities about designing other antibody bound active CRC chemotherapeutics to increase on-target effect and minimize systemic toxicity.

24. Bacac M, Klein C, Umana P, et al. CEA TCB: a novel head-to-tail 2:1 T cell bispecific antibody for treatment of CEA-positive solid tumors. Oncoimmunology 2016;5(8):e1203498. [PubMed: 27622073]

25•. Tabernero J, Melero I, Ros W, et al. Phase Ia and Ib studies of the novel carcinoembryonic antigen (CEA) T cell bispecific (CEA CD3 TCB) antibody as a single agent and in combination with atezolizumab: Preliminary efficacy and safety in patients with metastatic colorectal cancer (mCRC). J Clin Oncol 2017;35(15):S3002.This trial highlights a novel approach to creating immunogenicity in the CRC tumor microenvironment by drawing T cells to CEA expressing cells. It also demonstrates preliminary potential for utilizing bi-specific $\mathrm{T}$ cell antibodies in conjunction with checkpoint inhibitors to augment a cytotoxic $\mathrm{T}$ cell response.

26. Troiani T. RAS in colorectal cancer: ESMO biomarker factsheet http://oncologypro.esmo.org/ Education-Library/Factsheets-on-Biomarkers/RAS-in-Colorectal-Cancer.

27. Ajdei A. Blocking oncogenic Ras signaling for Cancer therapy. J Natl Cancer Inst 2001;14(18): 1062-74.

28. Corcoran R, Cheng K, Engelman J. Synthetic lethal interaction of combined BCL-XL and MEK inhibition promotes tumor regressions in KRAS mutant cancer models. Cancer Cell 2013;23(1): 121-8. [PubMed: 23245996]

29. O’Hara M, Edmonds C, Farwell M, et al. Phase II pharmacodynamic trial of palbociclib in patients with KRAS mutant colorectal cancer. J Clin Oncol 2015;33(3):S626.

30. Maitra R, Seetharam R, Tesfa L, et al. Oncolytic reovirus preferentially induces apoptosis in KRAS mutant colorectal cancer cells, and synergizes with irinotecan. Cancer Cell 2013;23(1):121-8. [PubMed: 23245996]

31. Gorlick R, Banerjee D. Fluoropyrimidine resistance in colon cancer. Expert Rev Anticancer Ther 2002;2(4):409-16. [PubMed: 12647984]

32. Ghazaly E, Slusaczyk M, McGuigan C, et al. NUC-3373: a novel pyrimidine nucleotide analogue that overcomes key cancer drug resistance limiting patient survival. Mol Cancer Ther 2015;14(12):S2.

33. Song Z, Wei B, Lu C, Li P, Chen L. Glutaminase sustains cell survival via the regulation of glycolysis and glutaminolysis in colorectal cancer. Oncol Lett 2017;14:3117-23. [PubMed: 28928849]

34. Ren W, Liu G, Yin J, Tan B, Wu G, Bazer FW, et al. Amino-acid transporters in T-cell activation and differentiation. Cell Death Dis 2017;8(3):e2655. [PubMed: 28252650]

35. Parikh K, Cang S, Sekhri A, et al. Selective inhibitors of nuclear export (SINE) — a novel class of anti-cancer agents. J Hematol Oncol 2014;7(78):1-8. [PubMed: 24387695]

36. Chung H, Fragomeni R, Shacham S, et al. Use of selective inhibition of nuclear export (SINE) using a CRM1/XPO1 antagonist to overcome resistance to CPT-11 in colon cancer in preclinical models. J Clin Oncol 2013;31(4):S396.

37. Mau-Soerensen M, Razak A, Mahipal A, et al. Safety and antitumor activity of selinexor (KPT-330), a first-in-class, oral XPO1 selective inhibitor of nuclear export: a phase I study expanded with colon cancer cohort. J Clin Oncol 2014;32(3):S482.

38. Novellasdemunt L, Antas P, Li VSW. Targeting Wnt signaling in colorectal cancer. A review in the theme: cell signaling: proteins, pathways and mechanisms. Am J Phys Cell Phys 2015;309(8):C511-21. 10.1152/ajpcell.00117.2015. 
39. Bahrami A, Amerizadeh F, Shahidsales S, et al. Therapeutic potential of targeting Wnt/ $\beta$-catenin pathway in treatment of colorectal cancer: rational and progress. J Cell Biochem 2017;118:1979_ 83. [PubMed: 28109136]

40. Hubbard JM, Grothey A. Napabucasin: an update on the first-in-class cancer stemness inhibitor. Drugs 2017;77:1091-103. [PubMed: 28573435]

41. Larson T, Ortuzar F, Bekaii-Saab T, et al. BBI608-224: a phase Ib/II study of cancer stemness inhibitor napabucasin (BBI-608) administered with panitumumab in KRAS wild-type patients with metastatic colorectal cancer. J Clin Oncol 2017;35(4):S677.

42•. Bendell J, Hubbard JM, O’Neill B, et al. Phase 1b/II study of cancer stemness inhibitor napabucasin (BBI-608) in combination with FOLFIRI +/- bevacizumab (bev) in metastatic colorectal cancer (mCRC) patients (pts). J Clin Oncol 2017;35(15):S3529. This study highlights the promise of napabucasin to reverse chemosensitivity for mCRC patients who have progressed previously on agents such as fluoropyrimidines and irinotecan. Rates of overall response and disease control were nearly identical between patients who were FOLFIRI-exposed or FOLFIRInaïve.

43. Grothey A, Shah M, Yoshino T, et al. CanStem303C trial: a phase III study of napabucasin (BBI-608) in combination with 5-fluorouracil (5-FU), leucovorin, irinotecan (FOLFIRI) in adult patients with previously treated metastatic colorectal cancer (mCRC). J Clin Oncol 2017;35(15):S3619.

44•. Shitara K, Yamanaka T, Denda T, et al. Reverce: randomized phase II study of regorafenib followed by cetuximab versus the reverse sequence for metastatic colorectal cancer patients previously treated with fluoropyrimidine, oxaliplatin, and irinotecan. J Clin Oncol 2018;36(4):S557.This study suggests the order of when we utilize regorafenib may play a significant role in its effectiveness. Utilizing regorafenib prior to cetuximab and irinotecan in RAS WT patients improved OS markedly compared to using the drug after the combination.

45. Bekaii-Saab T, Ou FS, Anderson D, et al. Regorafenib dose optimization study (ReDOS): randomized phase II trial to evaluate dosing strategies for regorafenib in refractory metastatic colorectal cancer (mCRC): an ACCRU network study. J Clin Oncol 2018;36(4):S611. 


\section{Opinion statement}

Choosing the optimal treatment approach for patients with metastatic colorectal cancer (mCRC) demands that oncologists assess both clinical and genomic variables and individualize care based upon the findings. Clinically, choices depend on assessing the side of the colon in which the primary tumor originates, the sites and burden of metastatic disease, the patient's performance status, and their individual comorbidities. Genomic assessment of the tumor to discern the mutational status of genes such as RAS/RAF, HER2, and TRK, as well as assessing whether tumors have defective mismatch repair (dMMR) or high microsatellite instability (MSI-H), all factor in to potential treatment options and can determine clinical trial eligibility. Metastasectomy may be an option for patients with a low burden of disease and accessible liver- or lung-limited metastases. In some unresectable cases, systemic therapy with a FOLFOX- or FOLFIRI- based regimen with or without a biologic agent can lead to sufficient disease reduction to make a patient eligible for resection of metastatic disease. Tumor sidedness and $R A S$ mutational status guide which biologic we add to the initial chemotherapy backbone, with patients with left-sided, $R A S$ wild-type (WT) tumors receiving anti-epidermal growth factor receptor (EGFR)-directed therapy and patients with right-sided tumors or those with $R A S$ mutations receiving bevacizumab. In patients with tumors that manifest microsatellite instability or deficient mismatch repair, we typically administer checkpoint inhibitors such as pembrolizumab or nivolumab after progression on irinotecan- or oxaliplatinbased therapies. In patients with progressive disease, we routinely send tumor tissue for next generation sequencing (NGS) to assess for the presence of actionable genomic alterations such as $H E R 2, B R A F$, and TRK fusions and offer them the option of enrollment on clinical trials with agents targeting those or other identified alterations. 


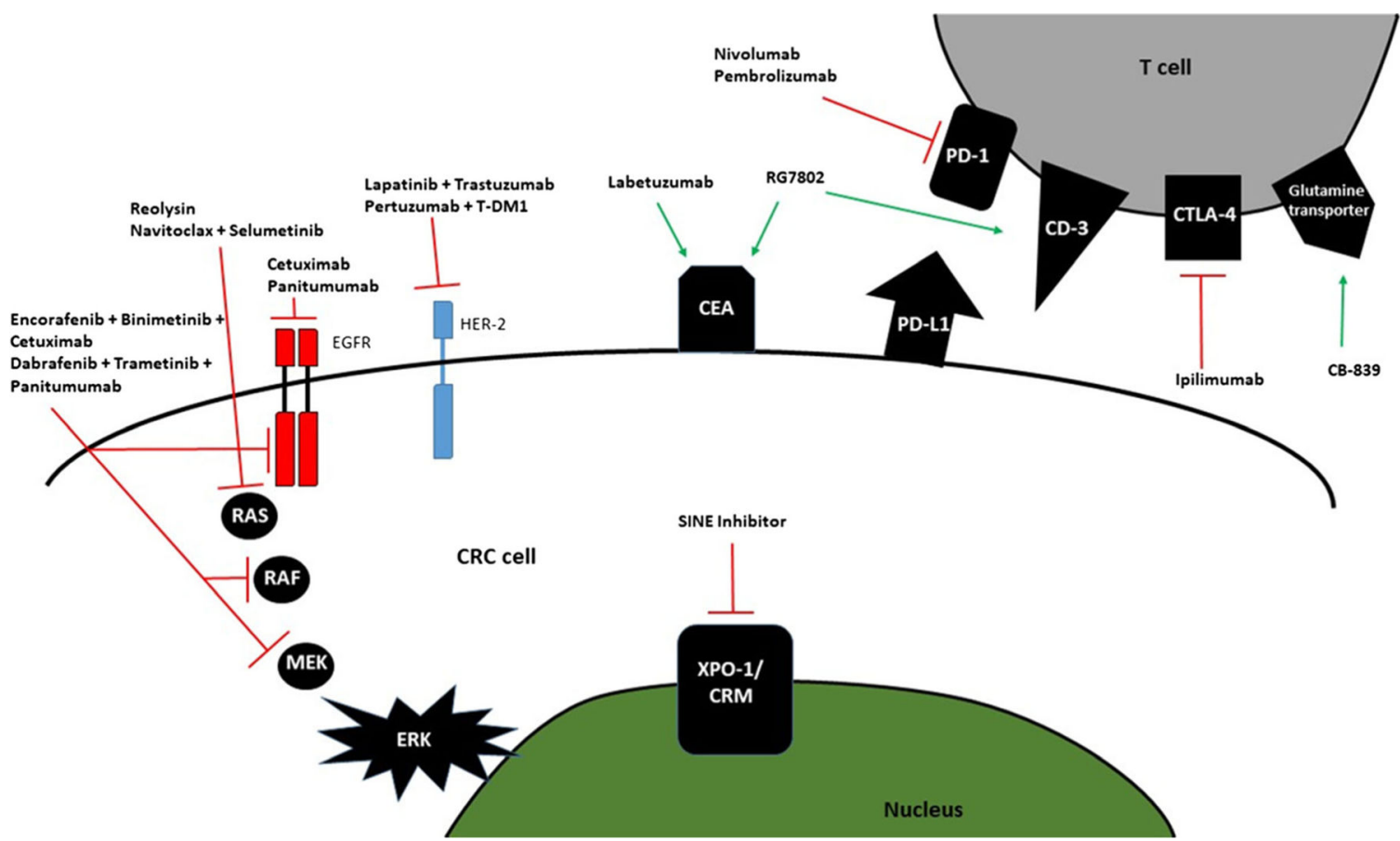

Fig. 1.

Schema of multiple pathway targets in CRC described in the review. Also illustrated is the T cell and its multiple points of interaction with CRC cells which can be exploited therapeutically. Arrows refer to agonistic binding while the "- 


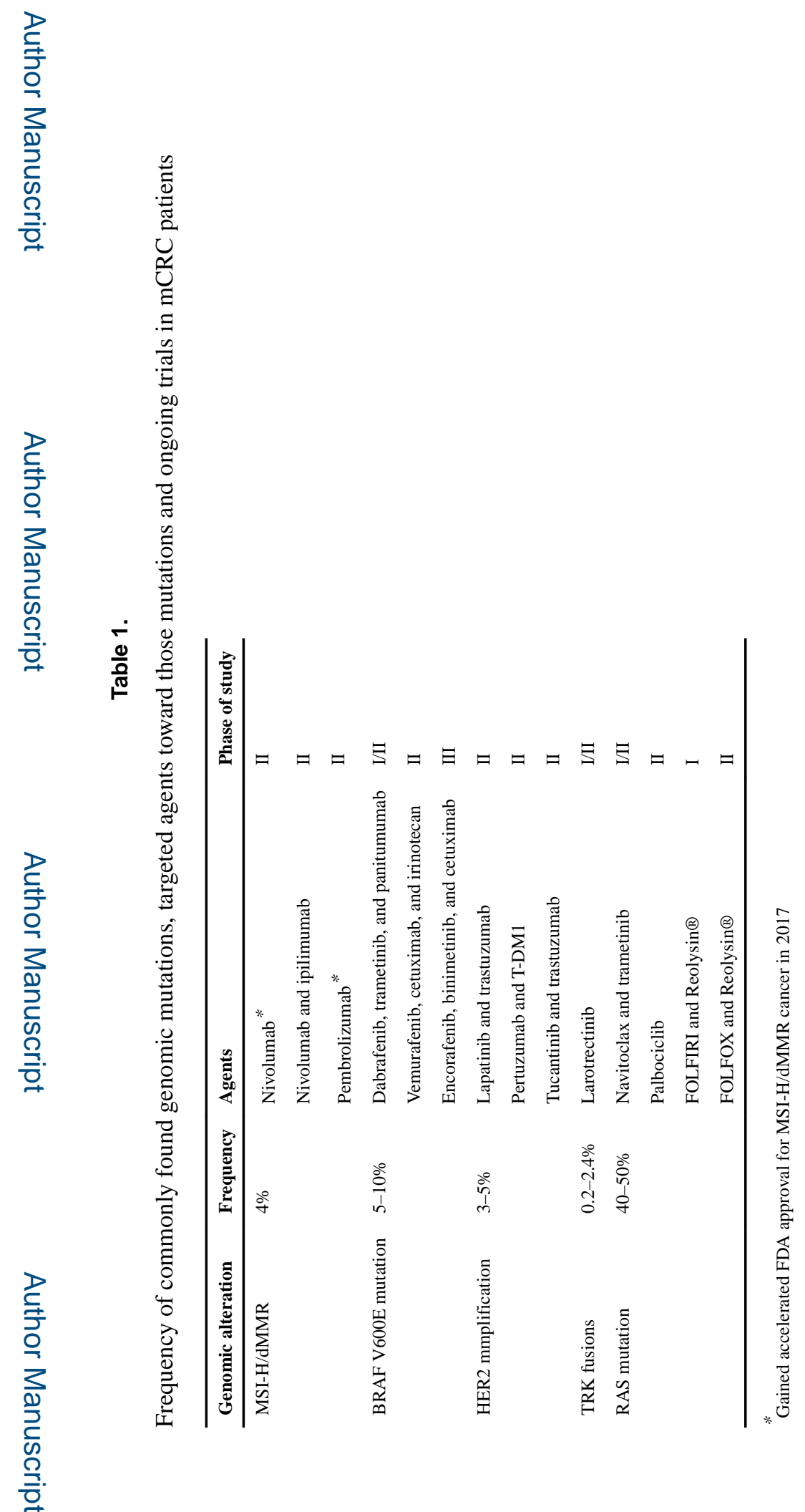

Curr Treat Options Oncol. Author manuscript; available in PMC 2019 May 04. 